Article

\title{
Accurate Mass Measurements for Planetary Microlensing Events Using High Angular Resolution Observations
}

\author{
Jean-Philippe Beaulieu 1,2 \\ 1 School of Physical Sciences, University of Tasmania, Private Bag 37 Hobart, Tasmania 7001, Australia; \\ jeanphilippe.beaulieu@utas.edu.au or beaulieu@iap.fr \\ 2 Sorbonne Universités, UPMC Université Paris 6 et CNRS, UMR 7095, Institut d'Astrophysique de Paris, \\ 98 bis bd Arago, 75014 Paris, France
}

Received: 5 March 2018; Accepted: 8 April 2018; Published: 24 April 2018

\begin{abstract}
The microlensing technique is a unique method to hunt for cold planets over a range of mass and separation, orbiting all varieties of host stars in the disk of our galaxy. It provides precise mass-ratio and projected separations in units of the Einstein ring radius. In order to obtain the physical parameters (mass, distance, orbital separation) of the system, it is necessary to combine the result of light curve modeling with lens mass-distance relations and/or perform a Bayesian analysis with a galactic model. A first mass-distance relation could be obtained from a constraint on the Einstein ring radius if the crossing time of the source over the caustic is measured. It could then be supplemented by secondary constraints such as parallax measurements, ideally by using coinciding ground and space-born observations. These are still subject to degeneracies, like the orbital motion of the lens. A third mass-distance relation can be obtained thanks to constraints on the lens luminosity using high angular resolution observations with $8 \mathrm{~m}$ class telescopes or the Hubble Space Telescope. The latter route, although quite inexpensive in telescope time is very effective. If we have to rely heavily on Bayesian analysis and limited constraints on mass-distance relations, the physical parameters are determined to $30-40 \%$ typically. In a handful of cases, ground-space parallax is a powerful route to get stronger constraint on masses. High angular resolution observations will be able to constrain the luminosity of the lenses in the majority of the cases, and in favorable circumstances it is possible to derive physical parameters to $10 \%$ or better. Moreover, these constraints will be obtained in most of the planets to be discovered by the Euclid and WFIRST satellites. We describe here the state-of-the-art approaches to measure lens masses and distances with an emphasis on high angular resolution observations. We will discuss the challenges, recent results and perspectives.
\end{abstract}

Keywords: exoplanets; planetary systems; microlensing; adaptive optics

\section{Introduction}

Gravitational microlensing is unique in its sensitivity to exoplanets in the Earth-Saturn mass range beyond the snow line [1-3], where the core accretion theory predicts that the most massive planets will form $[4,5]$. It gives us the opportunity to detect exoplanets orbiting host stars ranging from just over a solar mass down to the brown dwarf regime, and it can find them at distances ranging from a few hundred pc all the way to the central Galactic Bulge. Microlensing planets are detected without using any light from the planet host star. This allows planets to be discovered orbiting very faint stars (contrary to transit and radial velocity), but it can also make it difficult to infer the properties of the 
host stars. Over $\sim 90$ planets have been discovered by microlensing over the past 13 years ${ }^{1}$, including cold super Earths [6-10] cold Neptunes [11-13], Saturns in the disk [14,15], planets in the Bulge [16-19], multiple planet systems [20-23], a circumbinary planet [24], brown dwarf desert objects [25,26] brown dwarfs orbiting M dwarfs [27] and a first exomoon candidate [28]. Surprisingly a M dwarf orbited by a super Earth has been serendipitously found towards the anti-galactic center with a source star at 800 pc ([29]). Exoplanets could also be discovered in the M31 galaxy and a first candidate has been reported by [30].

Although the number of detected planets is relatively low compared to that discovered by the radial velocity and transit methods, microlensing probes a part of the parameter space (host separation vs. planet mass), which is mostly not accessible in the medium term to any other technique (Figure 1). Microlensing complements these detections because it is most sensitive to planets beyond the distance where water ice forms (the snow line), and down to earth mass planets.

Gould et al. [31] have made the first measurement of the frequency of ice and gas giants beyond the snow line, and have shown that this is about seven times higher than closer-in systems probed by the Doppler method. This comparison suggests that most giant planets do not migrate inwards very far. Howard et al. [32] have presented the first abundances of planets orbiting solar like stars within 0.25 AU using Kepler, while [33] have measured the abundance of Neptunes and super Earths using radial velocities. These studies show that $17-30 \%$ of solar like stars have planets on short orbits. With the microlensing technique, [34], measured the fraction of bound planets over 0.5-10 AU orbits from 10 Jupiter mass to 5 Earth mass and showed that planets around stars are the rule, rather than the exception. Then, [35], found high planet occurrence rates for the Kepler GK dwarf sample.

The MOA collaboration has recently measured the mass ratio function [36] beyond the snow line. This mass ratio function rises steeply toward lower masses as $q^{-0.85 \pm 0.13}$ down to $q \sim 10^{-4}$ where there is a break and possible peak in the mass ratio function. This is broadly consistent with the core accretion prediction that Neptune-Saturn ice giants should be more common than Jupiters, particularly in orbits around low-mass stars [37] but it shows that these population synthesis models under-predict the number of planets with planet:star mass ratios $q>10^{-4}$ as shown in Figure 1. Models from the Bern group [38] show a similar factor 10 discrepancy. These microlensing results are consistent with radial velocity and direct imaging surveys [39] but only microlensing probes the population of planets below Jupiter at these relatively wide separations on a routine basis. A major impediment to understanding this discrepancy with planet formation models is the fact that the microlens planets have been characterized only by their mass ratios, without knowledge of the host masses (see also [40]).

Indeed, the modeling of the observed planetary microlensing light curves gives the mass-ratio $q$ and projected separation $d$ in units of the angular Einstein radius $\Theta_{E}$ of the host star and its planet companion with an accuracy of $5-10 \%$ typically. Using maps from the OGLE-III collaboration we have a source distance $D_{S}$ estimate [41]. Unfortunately, there is only a single measurable parameter, the Einstein radius $\Theta_{E}$ crossing time, $t_{E}$, to constrain the lens mass $M_{L}$, distance $D_{L}$ and the relative lens-source proper motion, $\mu_{\text {rel }}$.

We have

$$
\Theta_{E}=1.01 \mathrm{mas}\left(\frac{M}{M_{\odot}}\right)^{1 / 2}\left(\frac{D_{S}-D_{L}}{D_{S} D_{L}} 8 \mathrm{kpc}\right)^{1 / 2}
$$

and

$$
t_{E}=1.01 \mathrm{yr}\left(\frac{M}{M_{\odot}}\right)^{1 / 2}\left(\frac{D_{S}-D_{L}}{D_{S} D_{L}} 8 \mathrm{kpc}\right)^{1 / 2}\left(\frac{1 \text { mas } \mathrm{yr}^{-1}}{\mu_{r e l}}\right)
$$

1 The Extrasolar Planets Encyclopaedia http:/ / exoplanet.eu/ and the NASA exoplanet archive, https:/ / exoplanetarchive. ipac.caltech.edu/. 
As a consequence, the physical mass and distance to the lens system are usually determined via a Bayesian analysis including a galactic model, and/or using additional constraints. There are three mass-distance relations that might constrain the physical parameters of the system, which can be combined for an accurate lens mass measurement. We are going to review them in the next sections.

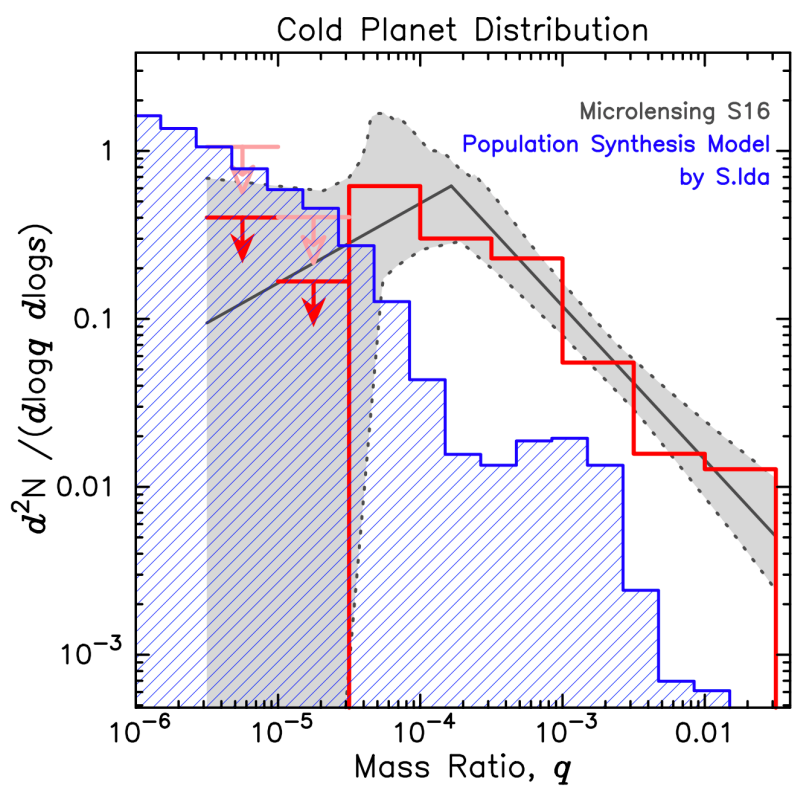

Figure 1. Microlensing mass ratio function [36] beyond the snow-line (in red) compared to the [5] core accretion population synthesis model (in blue). The population synthesis model underproduces wide separation planets by a factor of 10 for all mass ratios above the break at $q \sim 10^{-4}$. The mass ratio function is modeled with a broken power law with the grey shaded region indicating the $1 \sigma$ contours (courtesy of D. Bennett).

\subsection{Mass-Distance Relation from Resolving the Source by Caustics}

Fortunately, the majority of binary microlensing events have sharp light curve features that resolve the finite angular size of the source radius $\Theta_{*}$ crossing time to be measured in unit of the Einstein ring radius $\Theta_{E}$. Moreover, for a large fraction of the microlensing events we know the magnitude and color of the source. Therefore, we can use an infrared surface brightness relation, for example, [42] that links $(V-H, H)$ magnitudes to angular radius:

$$
\log \left(2 \Theta_{*}(\text { mas })\right)=0.536654+0.072703(V-H)-0.2 H
$$

This relation is a revision of the original [43], incorporating more data and excluding some unreliable measurements. We can then estimate the angular Einstein radius, $\Theta_{E}$, which is usually derived to $10 \%$ or better. This constraint then leads to a mass-distance relation between lens mass $M_{L}$ at distance $D_{L}$, under the form

$$
M_{L}=\frac{\theta_{E}^{2}}{\kappa \pi_{r e l}} \text { with } \pi_{\text {rel }}=\mathrm{AU}\left(\frac{1}{D_{L}}-\frac{1}{D_{S}}\right) \text { and } \kappa=8.144 \text { mas } M_{\odot}^{-1}
$$

\subsection{Mass-Distance Relation from Parallax Measurements}

A second mass-distance relation is obtained when the microlensing parallax, $\pi_{E}$, is measured [44]. This requires detecting the Earth orbital motion effect in the light curve for microlensing events with time scale larger than a month [8] or simultaneous observations of strong features from multiple sites at different latitudes [45]. An ideal configuration is to observe the microlensing event from two telescopes 
separated by $\sim 0.5+\mathrm{AU}$ [46-48]. Both configurations relate the projected size of the Einstein radius to solar system scales. This yields a relation between the parallax $\pi_{E}$ and the lens mass $M_{L}$,

$$
M_{L}=\frac{\theta_{E}}{\kappa \pi_{E}}
$$

This allows the elimination of $\theta_{E}$ to give a useful mass-distance relation for the case when we have well-defined parallax $\pi_{E}$ but unknown $\theta_{E}$ :

$$
M_{L}=\frac{\pi_{\mathrm{rel}}}{\kappa \pi_{E}^{2}}
$$

In practice, with ground-based data alone, $\pi_{E}$ is often not well constrained. Moreover, there is a degeneracy with the orbital motion of the planetary companion from the fitted light curve [15]. Even $\pi_{E}$ measurements from the Spitzer space telescope yield degenerate light curve models, but it is most of the time much more constrained than the ground-based. Figure 2 gives a superb example of a strong parallax signal detected between the ground and the Spitzer space telescope for the microlensing event OGLE 2014-BLG-0124 [49,50]. Good ground-space parallax observations combined with caustic crossings can give precise physical parameters for a system with a Neptune mass planet [13]. Shvartzvald et al. [10] combined Spitzer and ground-based Korea Microlensing Telescope Network measurements to detect an Earth mass planet orbiting an ultracool dwarf. Degeneracies of a system composed of a $\mathrm{G}$ dwarf orbited by a $13.4 \pm 0.9 M_{\text {Jupiter }}$ companion located in the galactic Bulge. Another route described by [51] is to use the parallax modulation during the orbit of a geosynchronous satellite to measure masses of low mass free-floating brown dwarfs or planets, without using ground-based data.

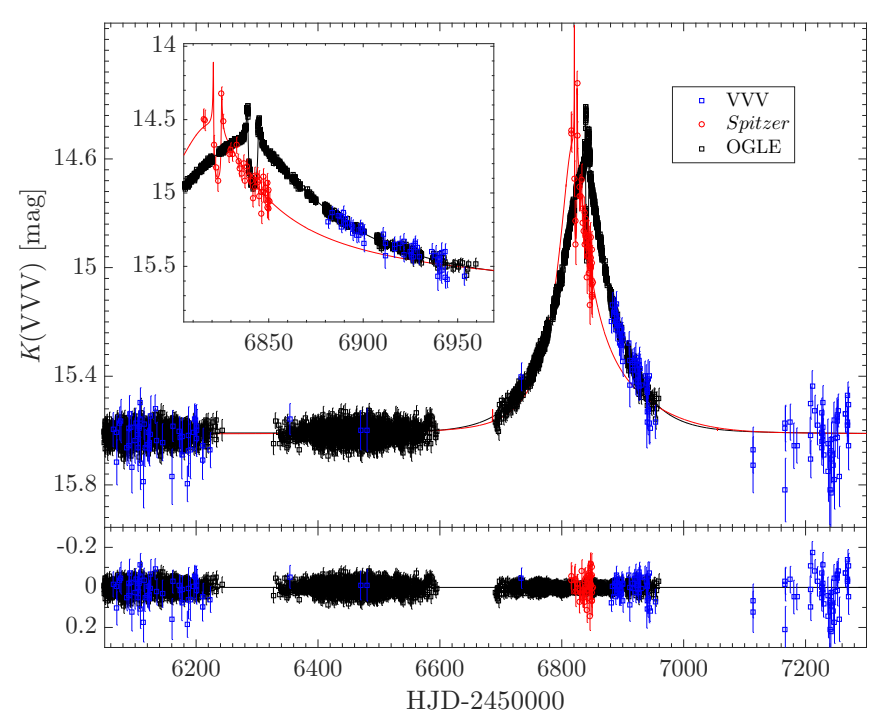

Figure 2. Light curve of the planetary model of OGLE-2014-BLG-0124 as observed from Earth by OGLE in I band (white) Vista $4 \mathrm{~m}$ at Paranal in $\mathrm{H}$ band (blue) and by Spitzer at $3.6 \mu \mathrm{m}$ (red), which was located 1 AU East of Earth in projection at the time of the observations. The Jovian planet appeared $\sim 20$ days earlier in Spitzer data compared to the ground. The mass ratio star/planet is well constrained from the modeling. The initial estimate of the mass of the host star is $\sim 0.7 M_{\odot} \mathrm{d}$ and the planet $\sim 0.5 M_{\text {jup }}$ (based on OGLE, Spitzer, [49]). The ground space-born parallax is giving an accurate mass-distance relation for the lens. We combined it with the mass-distance coming from high angular resolution observations to derive the physical parameters of the system [50]. 


\subsection{Mass-Distance Relation from Detecting Light from the Lens}

We have a third mass-distance relation if the flux from the lens system can be reliably measured. Using high angular resolution observations from adaptive optics on 8-10 m class telescopes or with the Hubble Space Telescope, it is possible to separate the contributions of the source and lens stars from other stars that are blended with the source in seeing-limited images ([52] and see Figure 3).

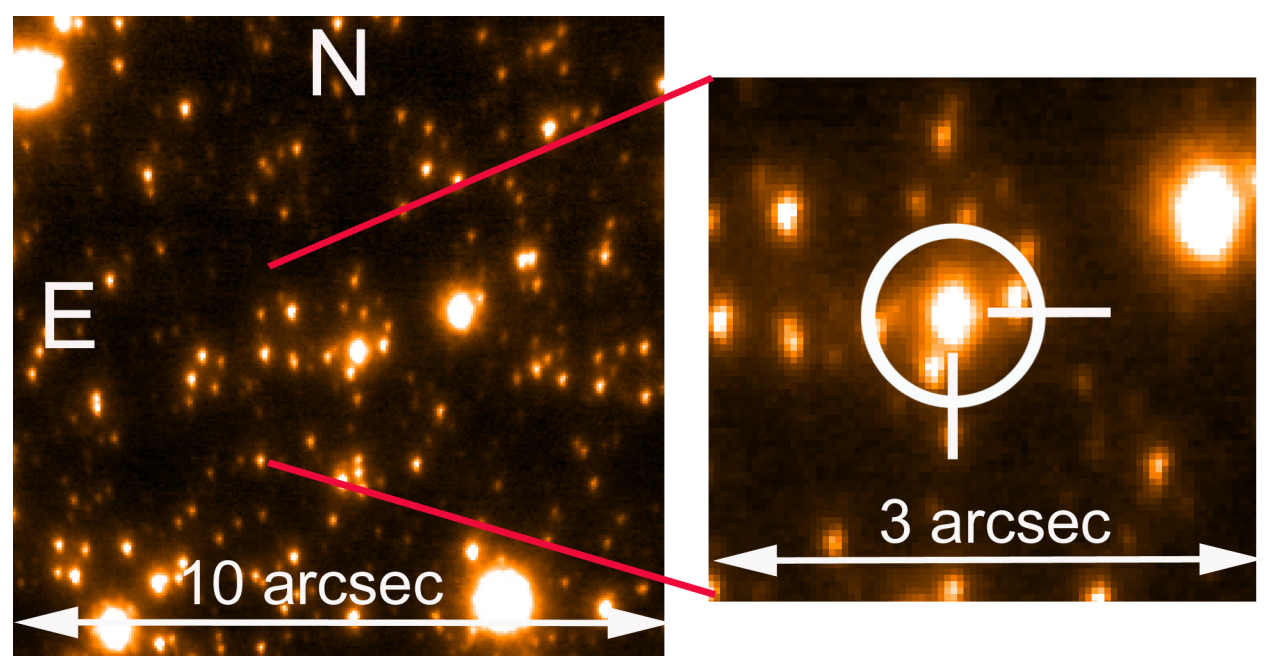

Figure 3. Keck II H-band observation of OGLE-2014-BLG-0124. The marked circle is 1 arcsec, showing that adaptive optics is essential for ground-based data to separate source and lens from unrelated blends. At the position of the source, we detect significant additional flux within 150 mas which is most likely the lens. The elongation seen on the image is consistent with the PSF shape of other nearby field stars [50].

We know the source luminosity from the light curve model, so we can subtract that from the measured flux which is a combination of source+lens flux. We combine this measurement with an empirical mass-luminosity relation [53] or isochrones [54] and then get an independent mass-distance relation as shown in Figures 4 and 5.

$$
M_{L}(\lambda)=10+5 \log \left(D_{L} / 1 k p c\right)+A_{L}(\lambda)+M_{\text {isochrone }}\left(\lambda, M_{L}, \text { age },[F e / H]\right)
$$

This has been done routinely on about $20+$ planetary microlensing events to date.

In this procedure, we made the assumption that the lens star itself is not variable. Only a fraction of a percent of main sequence stars towards the galactic bulge are variable with amplitude of $\sim 0.1$ mag or more. 


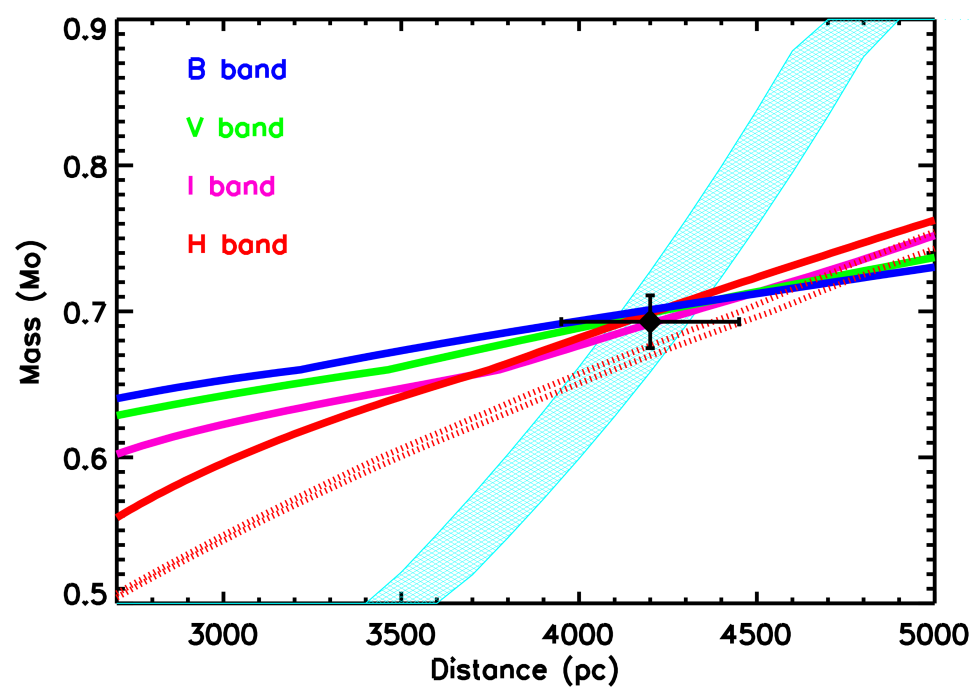

Figure 4. OGLE-2005-BLG-169 mass distance relations. The $\theta_{E}$ mass-distance relation is the cyan curve. The B, V, I, and $\mathrm{H}$ measured lens flux-distance relations, based on empirical mass-distance relations $[55,56]$ are plotted as blue, green, magenta, and red curves, respectively. Constraints from all passbands are in agreement and lead to $M_{L}=0.69 \pm 0.02 M_{\odot}$ orbited by a cold Neptune of $14.3 \pm 0.7 M_{\oplus}$. The system is at a distance $D_{L}=4.2 \pm 0.2 \mathrm{kpc}$. We also plotted the mass-distance relation using the $\mathrm{H}$ band measurement and 4-6.4 Gyr isochrones at solar metallicty [54]. If we would use this set of isochrones instead of the empircal one, we would obtain a mass $\sim 1 \sigma$ lower and a distance to the lens $\sim 0.5 \sigma$ shorter. It is required to clarify these small differences in the near future, for instance using a recalibrated empirical mass-luminosity relation using GAIA parallaxes.

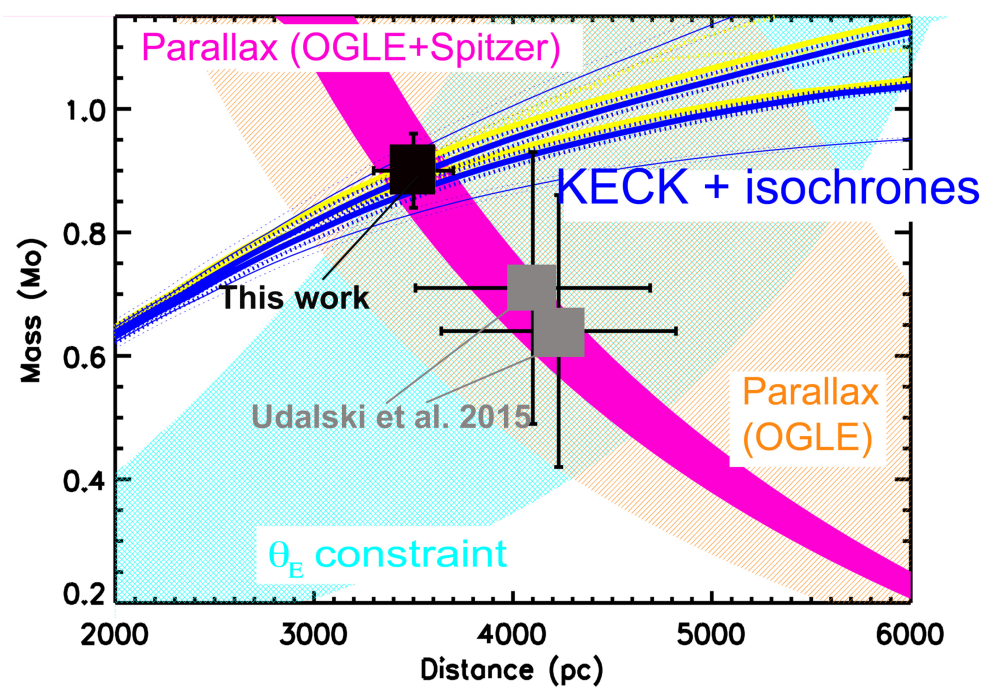

Figure 5. Mass-distance for the host star of the planetary microlensing OGLE-2014-BLG-0124. H-band isochrones in blue [54] for the $H_{L}=16.63 \pm 0.06$ lens brightness, while K-band isochrones for $H_{K}=16.44 \pm 0.06$ are plotted in yellow. The parallax mass-distance relation from OGLE alone is shown in orange, while the tight constraint from OGLE + Spitzer is plotted in magenta. The mass-distance from Einstein ring radius $\theta_{E}$ estimate is shown in cyan. In this particular case, there were no caustic crossings, so it led to a poor constrain from $\theta_{E}$. The grey squares mark the mass and distance estimates for the two solutions presented in the discovery paper [49], while the black square with its error bar is obtained after adding the KECK AO constraints [50]. 


\subsection{Contribution of Unresolved Light Aligned with the Source}

There is unfortunately a slight complication. When measuring the blended flux aligned with the source, does it come from the lens star alone? Or could some other star contribute additional flux within 50-100 mas of the source? When we detect blended light aligned with the source to better than 50-200 milliarcsecond PSF full-width at half-maximum, we must estimate if it is likely to be the lens star alone or if it has contributions from un-resolved additional stars:

- An ambient star, (aligned with source and lens not associated with either)

- a companion to the lens, (separated enough to not affect the lensing effect, while being unresolved)

- a companion to the source, (separated enough to not affect the lensing effect, while being unresolved).

This can be estimated statistically $[57,58]$, These two approaches differ in a number of details, but the final results are very close [50]. Currently, these corrections have been applied to a few systems [50,57-59] but it will have to be extended to all systems.

\subsection{Constraining Dark Lenses}

Microlensing is a unique method with sensitivity to free-floating planets and black hole candidates. High angular resolution observations permit to separate source + lens from unrelated subarcsecond blends. If no light excess is detected aligned with the source, we can put upper limits on the luminosity of the lens. The rigorous approach is that whether or not a small light excess is detected, a Bayesian analysis will be necessary to estimate what would be the most likely scenario: upper limit on the flux coming from the lens, luminous companion to the lens (separated enough from the lens to not affect the lensing effect, while being close enough in order not to be resolved), unrelated chance aligned star, unresolved companion to the source. For instance, for the microlensing MOA 2011-BLG-262 [28], measured the source+lens flux to $H_{S+L}=18.15 \pm 0.07$ while the two models were predicting $H_{S} \sim$ $18.22 \pm 0.04$. It is compatible with a dark lens and if we assign all potential flux to the lens, we can put an upper limit to $H_{L} \leq 21.06 \pm 1.0$. To summarize, high angular resolution observations will be an important diagnostic to be able to exclude false-alarm free-floating planets and black holes. However, because we will not detect any light from the lens, we cannot derive a mass-distance relation, only upper limits to the light coming from the lens. This has also been the case for the planetary microlensing OGLE-2007-BLG-387 [60] where an upper limit on the flux has been used as a constraint in the Bayesian analysis to estimate the host mass.

\subsection{Resolving Source and Lens and Measuring Relative Source-Lens Relative Proper Motion}

The relative source-lens proper motion is typically of the order of $\sim 5 \mathrm{mas} / \mathrm{yr}^{-1}$ and mostly in the range $\sim 1-10 \mathrm{mas} / \mathrm{yr}^{-1}$. Therefore, $5-10$ years after the microlensing event, it might be possible to directly resolve source and lens with adaptive optics on $10 \mathrm{~m}$ class telescopes. Then, we can measure the relative flux between the source and the lens, the amplitude and direction of the relative source lens proper motion. With the very stable PSF of the Hubble Space Telescope, it is possible to measure centroid variations while source and lens are not fully resolved, to contrain their flux ratio and relative proper motion [52].

Resolving lens and source and measuring their relative proper motion is done in a reference that is indistinguishable from the heliocentric reference frame. On the other hand, the contraint on the relative lens-source proper motion from the light curve is coming from the geocentric reference frame. We therefore have to convert our heliocentric relative proper motion measurement into the geocentric frame.

$$
\mu_{\text {rel,geo }}=\mu_{\text {rel,helio }}-\Delta \mu
$$

where

$$
\Delta \mu=\frac{\pi_{\mathrm{rel}} V_{\oplus, \perp}}{\mathrm{AU}}=\left(\frac{1}{D_{L}}-\frac{1}{D_{S}}\right) V_{\oplus, \perp} \quad \text { Dong et al. } 2009 .
$$




$$
\pi_{\text {rel }}=\operatorname{AU}\left(\frac{1}{D_{L}}-\frac{1}{D_{S}}\right)
$$

Here $V_{\oplus, \perp}$ is the velocity of the Earth projected on the sky at the time of the microlensing event (i.e., at $t_{0}$ ), in North and East coordinates [61]. We need to assume a starting value for the distance of the lens, $D_{L, 0}$, since the offset between them is a function of the relative distance between the lens and the source: Since the initial $D_{L, 0}$ value that we use to determine $\mu_{\text {rel,geo }}$ is a guess, we need to perform several loops to converge to a stable lens distance solution. For each tested value of $\mu_{\text {rel,helio, we obtain }}$ a value of $\mu_{\text {rel,geo }}$ that gives $\theta_{E}=\mu_{\text {rel,geo }} t_{E}$.

To date, resolving source and lens have been published on two microlensing events. Alcock et al. [62] resolved the disk lens and its Magellanic Cloud source in the microlensing event MACHO-LMC-5. Using HST and KECK [55,56], resolved source and lens in the planetary microlensing OGLE-2006-BLG-169 (Figure 6).
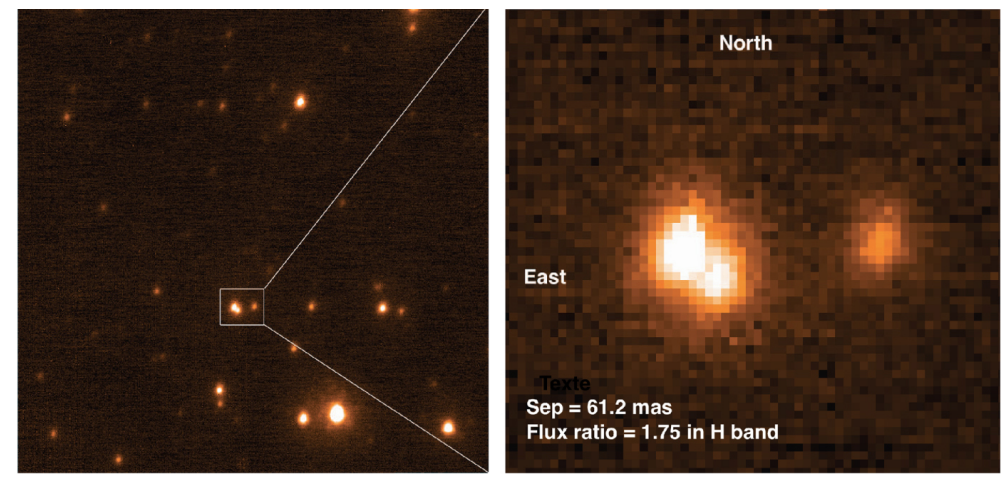

Figure 6. Left KECK H-band image of the source and lens stars in the planetary microlensing event OGLE-2005-BLG-169 (24 × 21 arcsec). Right, a zoom on the target showing the lens on the upper left and the source. They are separated by 61 mas. The extra star on the right was part of the measured blending in the ground-based microlensing light curve. We confirm the initial model of a Neptune mass planet orbiting a $\mathrm{K}$ dwarf [63] and improve the parameters of the system to better than $10 \%$ [55]. This is the first planetary microlensing where lens and source have been resolved by high angular imaging. Source and lens have also been resolved by HST [56].

\section{A Recipe for Constraining the Light Coming from the Lens with Adaptive Optics}

The objective is first to perform JHK photometry in crowded galactic bulge field for targets that are usually in the range $\mathrm{H}=15-19$. In practise we will be able to reach a precision of a few percent. Knowing the source flux, we aim to constrain a super-imposed lens flux thanks to the measurement. It might be difficult if the source is a giant with a main-sequence dwarf lens at few kpc; it will be much easier if the source and the lens have comparable brightness.

(1) Accurate position of the source star. Using the follow-up data is important to refine the position of the source star. It was done while the star was amplified to minimise potential contribution by blends. Typically with OGLE images we reach $\sim 20$ mas precision.

(2) Calibration data in JHK. Images collected by the VISTA $4 \mathrm{~m}$ telescope as part of the VVV survey are excellent [64] but the current interface and data products are not satisfactory. Our approach is to download all VVV data and develop tools to extract data cubes of 6 arcmin centred on the target. Then we perform PSF photometry using PSFEx [65] and SExtractor [66] from the AstrOmatic.net suite of tools. We then calibrate the catalogues using 2MASS both for astrometry and photometry the JHK data.

(3) Source estimates in $\mathbf{H}$ and/or K. If the microlensing target has been observed in IR, we extract its photometric light curve. The objective is to have measurements at different levels of amplification to be able to estimate accurately the source in IR bands. In the absence of such IR data, knowing 
the fitted V, I color of the source and extinction, we derive the color of the source and predict what would be its $\mathrm{H}$ and $\mathrm{K}$ magnitudes. It is better to stick closer to the observations and if possible, we prefer to avoid the color-transformation step.

(4) Preparing AO observations. First, we have to identify if there are some close stars bright enough to be acting as a natural guide star. They ideally have to be close to the target, 10-15 arcsec at most, while being bright enough to be able to close the adaptive optics loop and obtain diffraction limited images. In practise, it has been possible to use natural guide star for about half of the microlensing targets. We also have to identify stars for tip-tilt correction, but they can be fainter and further away. In the absence of bright natural guide star, it is possible to use at some telescopes (KECK) a laser guide star. We advise to build finding charts (with dimensions and orientation indicated) in the optical and also in the infrared, to be able to identify the field, guide-star, tip-tilt star and target quickly. Because galactic bulge fields are too crowded, we recommend dedicated observations of a dark field to estimate the sky.

(5) Observing strategy for constraining the lens flux. Any information at the subarcsecond level is valuable, even if the conditions are not excellent. Therefore, such measurement can be attempted in any decent observing conditions. In the case of KECK, we would advise to use the WIDE camera $(40$ arcsec) in order to have $\sim 100+$ VVV stars in the field for calibration. We advise a number of dithered observations, with a step of few arcsec. It should be kept in mind that such cameras usually have fairly large distortion.

(6) Resolving lens and source, measuring the relative proper motion. With very stable PSF as achieved with HST, it is possible to constrain the relative source/lens flux and relative proper motion while not resolving them, as done by [56]. It can be done even if source and lens are separated only a fraction of the FWHM. Given the adaptive optics of PSF stability, we are currently limited to resolving source and lens with a separation of $\sim 60+$ mas. The measurement will be done using for instance the NARROW camera at KECK (10 arcsec field of view). It can be attempted only under best observing conditions, when 45-60 mas images are being obtained.

(7) Data processing. First, it is necessary to correct for the dark current and flat-fields in a standard way. We also need to observe a field with very few or no stars to estimate the sky contribution in the observations. A possibility is to target a dark cloud located about 400 arcsec north and 713 arcsec west of the Center. We then correct the science images. We then correct for the distortion of the images, using accurate maps provided by the observatory for the camera. We then compute an astrometric solution for each image using VVV as a reference and SCAMP [67]. Images are re-gridded and then stacked using SWARP [68].

(8) Data analysis. We perform aperture photometry using the SExtractor package. We then cross-identify the stars observed both by AO and VVV, for example using TOPCAT [69]. We usually are able to calibrate to $1 \%$ routinely with the KECK WIDE Camera. With a smaller field of view, it could become tricky since few stars at best might be available, and there is always the risk that one is variable, or that nearby bright stars are contaminating the photometry and making it impractical. Unless we attempt to resolve blended source and lens, we perform aperture photometry to measure the fluxes. It is also possible to perform PSF fitting using some tools such as AIROPA [70].

\section{Getting the Mass and Distance to the Lens}

\subsection{Calculating Extinction to the Source and the Lens}

Nataf et al. [41] combined wide field imager data from OGLE and VVV survey of the Galactic Bulge [64,71]. Knowing the mean properties of the red-clump stars, they estimated distances and extinctions over 4000 line of sights. By interpolating these measurements, it is possible to obtain distance, extinction maps and coefficients for the galactic Bulge. A convenient server has been developed by OGLE based on [41] analysis. It should be noted that the extinction towards the Bulge is so called "anomalous", and that the coefficients differ from the ones in the solar neighbourhood [72]. 
It is also possible to use multi-band photometry to build color magnitude diagrams and derive extinction and distances for the analyzed fields.

In practise, we adopt as a scale height of the dust towards the galactic bulge $\tau_{\text {dust }}=$ $(0.120 \mathrm{kpc}) / \sin (b)$, where $b$ is the galactic latitude. Then we write the lens extinction in $\mathrm{H}$ band $\mathrm{A}_{H_{L}}$ :

$$
A_{H_{L}}=\left(1-e^{-D_{L} / \tau_{\text {dust }}}\right) /\left(1-e^{-D_{S} / \tau_{\text {dust }}}\right) A_{H_{S}} .
$$

This model attributes most of the extinction to short distances. Another route is to perform additional observations (such as using X-shooter on the VLT) to actually fit the UV-to-NIR spectra energy distribution and extinction simultaneously [73].

\subsection{Using Empirical Mass-Luminosity Relations or Isochrones}

Thanks to the high angular resolution images, we have estimates of the luminosity of the lens. We then adopt a set of isochrones or mass-luminosity relations, and then, using Equation (4) we obtain a mass-distance relation. Figure 4 is showing the BVIH constraints on the host star of the microlensing event OGLE-2005-BLG-169. The empirical mass-luminosity relation calibrated using stars from the solar neighbourhood is giving masses systematically higher than the isochrones from [54] for a solar metallicity by a few percent. We are now reaching the level of precision where this will have to be taken into account. GAIA parallaxes will provide a good opportunity to recalibrate these mass-luminosity relations and hopefully remove the remaining small discrepancies in mass-luminosities relations.

\subsection{Current Limitations}

We can identify several steps in the process that could be improved. First, the distance to the source is now becoming one of the dominant sources of error. Revisiting the galactic Bulge structure using constraints from GAIA combined with multiband photometry is necessary. Secondly, extinction, especially for the low latitude fields should be revisited. Indeed, the first studies of the fields in the galactic plane with $A_{K} \sim 1.5$ [74], shows the limitation of the simple model presented in Section 3.1 and suggest that the nature of the dust is different. This leads to ambiguous results in these particular fields, and is also the one with the highest optical depth to microlensing and preeminent line of sight for Euclid and WFIRST.

\section{Results}

High angular resolution observations have been obtained with KECK, SUBARU, VLT or MAGELLAN on about 40 planetary microlensing events and 15 free-floating planets, while the Hubble Space Telescope observed six planetary microlensing to date. Such observations are relatively cheap in telescope time, since it requires about one hour with overheads for the AO observations, for instance. Nevertheless, they are a powerful way to constrain the planetary systems after their discoveries. The results from Table 1 show a significant improvement in the mass and distance measurements of the host stars. Contrary to popular belief, it is also clear that once the microlensing event is over, it is still possible to perform additional observations to refine the physical parameters of the system.

The next step is to extend this study to the entire [36] sample, and then be able to plot a mass-function instead of the mass-ratio function (Figure 1). It will also be possible to study the spatial distribution and discuss the relative abundance in the disk and in the Bulge of our galaxy. 
Table 1. Lens host masses and distances of planetary systems discovered by microlensing with constraints from high angular resolution constraints. We list the initial estimate if it was without high angular resolution with the label "initial". If the initial study included high angular resolution it is then listed only with the label "with HR". Otherwise, the HR labels indicate the current best estimates for mass and distances for these host stars. (124) [49,50], (220) [59,75], (132) [58,76], (026) [23,77], (293) [17,78], (319) [14,59], (400) [59,79], (192) [7,9], (169) [55,56,63], (071) [80,81], (235) [52,59,82], (227) [57], (605) [12], (950) [83], (563) [84], (006) [85], (310) [16,18], (368) [11], (387) [60], (349) [24], (109) $[20,21]$.

\begin{tabular}{lrrrrr}
\hline Name & $\begin{array}{r}\text { Distance (kpc) } \\
\text { Initial }\end{array}$ & $\begin{array}{r}\text { Distance (kpc) } \\
\text { with HR }\end{array}$ & $\begin{array}{r}\left.\text { Mass } \mathbf{M}_{\odot}\right) \\
\text { Initial }\end{array}$ & $\begin{array}{r}\text { Mass }\left(\boldsymbol{M}_{\odot}\right) \\
\text { with HR }\end{array}$ & ref \\
\hline OGLE-2014-BLG-124 & $4.23 \pm 0.6$ & $3.5 \pm 0.2$ & $0.65 \pm 0.22$ & $0.90 \pm 0.05$ & $(124)$ \\
MOA-2013-BLG-220 & $1.6-6.5$ & $6.55 \pm 0.5$ & $0.05-0.8$ & $0.96 \pm 0.07$ & $(220)$ \\
OGLE-2013-BLG-132 & $3.9_{-1.3}^{+1.5}$ & $3.45 \pm 0.75$ & $0.54_{-0.23}^{+0.30}$ & $0.50 \pm 0.08$ & $(132)$ \\
OGLE-2012-BLG-026 & $4.08 \pm 0.4$ & $4.0 \pm 0.3$ & $0.82 \pm 0.13$ & $1.06 \pm 0.05$ & $(026)$ \\
MOA-2011-BLG-293 & $7.15 \pm 0.94$ & $7.72 \pm 0.44$ & $0.59_{-0.29}^{+0.35}$ & $0.86 \pm 0.06$ & $(293)$ \\
MOA-2009-BLG-319 & $6.1_{-1.2}^{+1.1}$ & $6.9 \pm 0.3$ & $0.38_{-0.18}^{+0.34}$ & $0.82 \pm 0.05$ & $(319)$ \\
MOA-2007-BLG-400 & $5.8_{-0.7}^{+0.8}$ & $7.2 \pm 0.5$ & $0.30_{-0.12}^{+0.19}$ & $0.95 \pm 0.06$ & $(400)$ \\
MOA-2007-BLG-192 & $1.0 \pm 0.4$ & $0.66_{-0.07}^{+0.1}$ & $0.06_{-0.021}^{+0.028}$ & $0.084_{-0.012}^{+0.015}$ & $(192)$ \\
OGLE-2005-BLG-169 & $2.7_{-1.3}^{+1.6}$ & $4.2 \pm 0.2$ & $0.49_{-0.29}^{+0.23}$ & $0.69 \pm 0.02$ & $(169)$ \\
OGLE-2005-BLG-071 & $1.5-5$ & $3.74_{-0.55}^{+0.68}$ & $0.08-0.5$ & $0.201_{-0.045}^{+0.053}$ & $(071)$ \\
OGLE-2003-BLG-235 & $5.2_{-2.9}^{+0.2}$ & $5.2 \pm 0.5$ & $0.36_{-0.28}^{+0.03}$ & $0.56 \pm 0.04$ & $(235)$ \\
\hline MOA-2016-BLG-227 & & $6.5 \pm 1.0$ & & $0.29_{-0.15}^{+0.23}$ & $(227)$ \\
OGLE-2013-BLG-605 & & $3.74_{-0.55}^{+0.68}$ & & $0.201_{-0.045}^{+0.053}$ & $(605)$ \\
OGLE-2012-BLG-950 & & $3.0_{-1.1}^{+0.8}$ & & $0.56_{-0.16}^{+0.12}$ & $(950)$ \\
OGLE-2012-BLG-563 & & $1.3_{-0.8}^{+0.6}$ & & $0.34_{-0.20}^{+0.12}$ & $(563)$ \\
OGLE-2012-BLG-006 & & $5.3_{-1.3}^{+0.8}$ & & $0.49_{-0.23}^{+0.27}$ & $(006)$ \\
MOA-2008-BLG-310 & & $7.7 \pm 1.1$ & & $0.21 \pm 0.14$ & $(310)$ \\
OGLE-2007-BLG-368 & & $5.9_{-1.4}^{+0.9}$ & & $0.64_{-0.26}^{+0.21}$ & $(368)$ \\
OGLE-2007-BLG-387 & & $7.2 \pm 0.8$ & & $0.11_{-0.06}^{+0.21}$ & $(387)$ \\
OGLE-2007-BLG-349 & & $2.76 \pm 0.38$ & & $0.41 \pm 0.07$ & $(349)$ \\
OGLE-2006-BLG-109 & & $1.51_{-0.12}^{+0.11}$ & & $0.30 \pm 0.07$ & \\
\hline & & & & $0.51_{-0.04}^{+0.05}$ & $(109)$ \\
\hline
\end{tabular}

Most microlensing planets are currently detected by combining high cadence observations obtained with wide field imager (OGLE-IV, MOA-II, KMTNeT, WISE, ROME-REA). Targeted observations of high magnification events with high sensitivity to exoplanets are done by follow up telescopes such at the $1.3 \mathrm{~m}$ Harlingten telescope at University of Tasmania Greenhill Observatory and the telescopes of the Las Cumbres Observatory. These observations are still valuable to be able to unveil a planetary anomaly such as in OGLE-2015-BLG-1395 [86], to well characterise a caustic crossing missed by survey telescope or for Terrestrial parallax [28]. Now, the sensitivity extends to $\sim 1$ Earth mass planets at the snow line in favorable cases [10].

There has been an aggressive campaign with the Spitzer space telescope to provide excellent ground-space parallaxes in the period 2014-2018 for a handful of planetary events [10,13,49]. First, the target has to be observable within a one month window of Spitzer and it has to be bright enough. Secondly, it is possible to do only weekly updates of observing targets. Because of the very nature of microlensing events, it is hard to predict early which ones will have a high planet detection efficiency. Ideally, it is much more efficient to update the observing strategy and target list on a daily basis at least. Updating every week the Spitzer target list means that some interesting targets will be missed, while observing time will be dedicated to targets that will turn out to have low interest. Moreover, Spitzer will have to concentrate on relatively bright targets. Given these constraints, the team for the Spitzer observations has set up a strategy to try to optimise their observing strategies [10]. Third, the parallax has to be well enough constrained by the observations. See [87] for the strategy and [88] for 
the post-2018 perspectives. The campaign 9 of Kepler-K2 gave only one planet [57] and no free-floating planets. The photometry with such large pixels in the Galactic Bulge has been more challenging than anticipated.

Despite these efforts on the parallax front, there is still a limitation on the accuracy of the determination of the physical parameters, that could be overcome with constraints from high angular resolution observations. The key ingredients required are infrared photometry high angular resolution observations from 8+m class telescopes and/or with V, I on the Hubble Space telescope. Recently, 10 nights have been allocated as a "Keck Key Strategic Mission Support" to observe all known microlensing planets to date. Within the next three years, we will obtain accurate mass measurements of $\sim 100+$ cold planets. We will then derive the cold planet mass-function and compare the relative planet populations in the disk and the bulge. While we will be conducting this work, we will also test the method that will be used routinely on WFIRST to measure masses for over $90 \%$ of the planets to be discovered by microlensing.

Acknowledgments: This work was supported by the University of Tasmania through the UTAS Foundation and the endowed Warren Chair in Astronomy. The author acknowledges his long term collaborators and in particular Virginie Batista (Paris), David Bennett (Goddard), Andrew Cole (UTAS), Jean-Baptiste Marquette (IAP). He acknowledges the financial support of Programme National de Planétologie (CNRS, INSU), PERSU from Sorbonne-Université and the CNES. Data presented herein were obtained at the W. M. Keck Observatory from telescope time allocated to the National Aeronautics and Space Administration through the agencys scientic partnership with the California Institute of Technology and the University of California. The Observatory was made possible by the generous financial support of the W. M. Keck Foundation. This publication makes use of data products from the Two Micron All Sky Survey, which is a joint project of the University of Massachusetts and the Infrared Processing and Analysis Center/California Institute of Technology, funded by the National Aeronautics and Space Administration and the National Science Foundation.

Conflicts of Interest: The authors declare no conflict of interest.

\section{Abbreviations}

The following abbreviations are used in this manuscript:

AO adaptive optics observations

FWHM Ful Width at Half Maximum

HST Hubble Space Telescope

PSF Point Spread Function

VVV VISTA Variables in the Via Lactea, a large infrared survey done with the VISTA 4m telescope

\section{References}

1. Mao, S.; Paczynski, B. Gravitational microlensing by double stars and planetary systems. Astrophys. J. 1991, 374, L37-L40. [CrossRef]

2. Gould, A.; Loeb, A. Discovering planetary systems through gravitational microlenses. Astrophys. J. 1992, 396, 104-114. [CrossRef]

3. Bennett, D.P.; Rhie, S.H. Detecting Earth-Mass Planets with Gravitational Microlensing. Astrophys. J. 1996, 472, 660-664. [CrossRef]

4. Lissauer, J.J.; Stewart, G.R. Growth of planets from planetesimals. In Protostars and Planets III; Levy, E.H., Lunine, J.I., Eds.; University of Arizona Press: Tucson, AZ, USA, 1993; pp. 1061-1088.

5. Ida, S.; Lin, D.N.C. Toward a Deterministic Model of Planetary Formation. I. A Desert in the Mass and Semimajor Axis Distributions of Extrasolar Planets. Astrophys. J. 2004, 604, 388-413. [CrossRef]

6. Beaulieu, J.P.; Bennett, D.P.; Fouqué, P.; Williams, A.; Dominik, M.; Jørgensen, U.G.; Kubas, D.; Cassan, A.; Coutures, C.; Greenhill, J.; et al. Discovery of a cool planet of 5.5 Earth masses through gravitational microlensing. Nature 2006, 439, 437-440. [CrossRef] [PubMed]

7. Bennett, D.P.; Bond, I.A.; Udalski, A.; Sumi, T.; Abe, F.; Fukui, A.; Furusawa, K.; Hearnshaw, J.B.; Holderness, S.; Itow, Y.; et al. A Low-Mass Planet with a Possible Sub-Stellar-Mass Host in Microlensing Event MOA-2007-BLG-192. Astrophys. J. 2008, 684, 663-683. [CrossRef] 
8. Muraki, Y.; Han, C.; Bennett, D.P.; Suzuki, D.; Monard, L.A.G.; Street, R.; Jorgensen, U.G.; Kundurthy, P.; Skowron, J.; Becker, A.C.; et al. Discovery and Mass Measurements of a Cold, 10 Earth Mass Planet and Its Host Star. Astrophys. J. 2011, 741, 22. [CrossRef]

9. Kubas, D.; Beaulieu, J.P.; Bennett, D.P.; Cassan, A.; Cole, A.; Lunine, J.; Marquette, J.B.; Dong, S.; Gould, A.; Sumi, T.; et al. A frozen super-Earth orbiting a star at the bottom of the main sequence. Astron. Astrophys. 2012, 540, A78. [CrossRef]

10. Shvartzvald, Y.; Yee, J.C.; Calchi Novati, S.; Gould, A.; Lee, C.U.; Beichman, C.; Bryden, G.; Carey, S.; Gaudi, B.S.; Henderson, C.B.; et al. An Earth-mass Planet in a 1 au Orbit around an Ultracool Dwarf. Astrophys. J. Lett. 2017, 840, L3. [CrossRef]

11. Sumi, T.; Bennett, D.P.; Bond, I.A.; Udalski, A.; Batista, V.; Domodellingik, M.; Fouqué, P.; Kubas, D.; Gould, A.; Macintosh, B.; et al. A Cold Neptune-Mass Planet OGLE-2007-BLG-368Lb: Cold Neptunes Are Common. Astrophys. J. 2010, 710, 1641-1653. [CrossRef]

12. Sumi, T.; Udalski, A.; Bennett, D.P.; Gould, A.; Poleski, R.; Bond, I.A.; Skowron, J.; Rattenbury, N.; Pogge, R.W.; Bensby, T.; et al. The First Neptune Analog or Super-Earth with a Neptune-like Orbit: MOA-2013-BLG-605Lb. Astrophys. J. 2016, 825, 112. [CrossRef]

13. Street, R.A.; Udalski, A.; Calchi-Novati, S.; Hundertmark, M.P.G.; Zhu, W.; Gould, A.; Yee, J.; Tsapras, Y.; Bennett, D.P.; RoboNet Project, T.; et al. Spitzer Parallax of OGLE-2015-BLG-0966: A Cold Neptune in the Galactic Disk. Astrophys. J. 2016, 819, 93. [CrossRef]

14. Miyake, N.; Sumi, T.; Dong, S.; Street, R.; Mancini, L.; Gould, A.; Bennett, D.P.; Tsapras, Y.; Yee, J.C.; Albrow, M.D.; et al. A Sub-Saturn Mass Planet, MOA-2009-BLG-319Lb. Astrophys. J. 2011, 728, 120. [CrossRef]

15. Bachelet, E.; Shin, I.G.; Han, C.; Fouqué, P.; Gould, A.; Menzies, J.W.; Beaulieu, J.P.; Bennett, D.P.; Bond, I.A.; Dong, S.; et al. MOA 2010-BLG-477Lb: Constraining the Mass of a Microlensing Planet from Microlensing Parallax, Orbital Motion, and Detection of Blended Light. Astrophys. J. 2012, 754, 73. [CrossRef]

16. Janczak, J.; Fukui, A.; Dong, S.; Monard, L.A.G.; Kozłowski, S.; Gould, A.; Beaulieu, J.P.; Kubas, D.; Marquette, J.B.; Sumi, T.; et al. Sub-Saturn Planet MOA-2008-BLG-310Lb: Likely to be in the Galactic Bulge. Astrophys. J. 2010, 711, 731-743. [CrossRef]

17. Batista, V.; Beaulieu, J.P.; Gould, A.; Bennett, D.P.; Yee, J.C.; Fukui, A.; Gaudi, B.S.; Sumi, T.; Udalski, A. MOA-2011-BLG-293Lb: First Microlensing Planet Possibly in the Habitable Zone. Astrophys. J. 2014, 780, 54. [CrossRef]

18. Bhattacharya, A.; Bennett, D.P.; Anderson, J.; Bond, I.A.; Gould, A.; Batista, V.; Beaulieu, J.P.; Fouqué, P.; Marquette, J.B.; Pogge, R. The Star Blended with the MOA-2008-BLG-310 Source Is Not the Exoplanet Host Star. Astron. J. 2017, 154, 59. [CrossRef]

19. Ryu, Y.H.; Yee, J.C.; Udalski, A.; Bond, I.A.; Shvartzvald, Y.; Zang, W.; Figuera Jaimes, R.; Jørgensen, U.G.; Zhu, W.; Huang, C.X.; et al. OGLE-2016-BLG-1190Lb: The First Spitzer Bulge Planet Lies Near the Planet/Brown-dwarf Boundary. Astron. J. 2018, 155, 40. [CrossRef]

20. Gaudi, B.S.; Bennett, D.P.; Udalski, A.; Gould, A.; Christie, G.W.; Maoz, D.; Dong, S.; McCormick, J.; Szymański, M.K.; Tristram, P.J.; et al. Discovery of a Jupiter/Saturn Analog with Gravitational Microlensing. Science 2008, 319, 927-930. [CrossRef] [PubMed]

21. Bennett, D.P.; Rhie, S.H.; Nikolaev, S.; Gaudi, B.S.; Udalski, A.; Gould, A.; Christie, G.W.; Maoz, D.; Dong, S.; McCormick, J.; et al. Masses and Orbital Constraints for the OGLE-2006-BLG-109Lb,c Jupiter/Saturn Analog Planetary System. Astrophys. J. 2010, 713, 837-855. [CrossRef]

22. Han, C.; Jung, Y.K.; Udalski, A.; Sumi, T.; Gaudi, B.S.; Gould, A.; Bennett, D.P.; Tsapras, Y.; Szymański, M.K.; Kubiak, M.; et al. Microlensing Discovery of a Tight, Low-mass-ratio Planetary-mass Object around an Old Field Brown Dwarf. Astrophys. J. 2013, 778, 38. [CrossRef]

23. Beaulieu, J.P.; Bennett, D.P.; Batista, V.; Fukui, A.; Marquette, J.B.; Brillant, S.; Cole, A.A.; Rogers, L.A.; Sumi, T.; Abe, F.; et al. Revisiting the Microlensing Event OGLE 2012-BLG-0026: A Solar Mass Star with Two Cold Giant Planets. Astrophys. J. 2016, 824, 83. [CrossRef]

24. Bennett, D.P.; Rhie, S.H.; Udalski, A.; Gould, A.; Tsapras, Y.; Kubas, D.; Bond, I.A.; Greenhill, J.; Cassan, A.; Rattenbury, N.J.; et al. The First Circumbinary Planet Found by Microlensing: OGLE-2007-BLG-349L(AB)c. Astron. J. 2016, 152, 125. [CrossRef] 
25. Street, R.A.; Choi, J.Y.; Tsapras, Y.; Han, C.; Furusawa, K.; Hundertmark, M.; Gould, A.; Sumi, T.; Bond, I.A.; Wouters, D.; et al. MOA-2010-BLG-073L: An M-dwarf with a Substellar Companion at the Planet/Brown Dwarf Boundary. Astrophys. J. 2013, 763, 67. [CrossRef]

26. Ranc, C.; Cassan, A.; Albrow, M.D.; Kubas, D.; Bond, I.A.; Batista, V.; Beaulieu, J.P.; Bennett, D.P.; Dominik, M.; Dong, S.; et al. MOA-2007-BLG-197: Exploring the brown dwarf desert. Astron. Astrophys. 2015, 580, A125. [CrossRef]

27. Bachelet, E.; Fouqué, P.; Han, C.; Gould, A.; Albrow, M.D.; Beaulieu, J.P.; Bertin, E.; Bond, I.A.; Christie, G.W.; Heyrovský, D.; et al. A brown dwarf orbiting an M-dwarf: MOA 2009-BLG-411L. Astron. Astrophys. 2012, 547, A55. [CrossRef]

28. Bennett, D.P.; Batista, V.; Bond, I.A.; Bennett, C.S.; Suzuki, D.; Beaulieu, J.P.; Udalski, A.; Donatowicz, J.; Bozza, V.; Abe, F.; et al. MOA-2011-BLG-262Lb: A Sub-Earth-Mass Moon Orbiting a Gas Giant Primary or a High Velocity Planetary System in the Galactic Bulge. Astrophys. J. 2014, 785, 155. [CrossRef]

29. Nucita, A.A.; Licchelli, D.; De Paolis, F.; Ingrosso, G.; Strafella, F.; Katysheva, N.; Shugarov, S. Discovery of a bright microlensing event with planetary features towards the Taurus region: A super-Earth planet. Mon. Not. R. Astron. Soc. 2018, 476, 2962-2967. [CrossRef]

30. Ingrosso, G.; Novati, S.C.; de Paolis, F.; Jetzer, P.; Nucita, A.A.; Zakharov, A.F. Pixel lensing as a way to detect extrasolar planets in M31. Mon. Not. R. Astron. Soc. 2009, 399, 219-228. [CrossRef]

31. Gould, A.; Dong, S.; Gaudi, B.S.; Udalski, A.; Bond, I.A.; Greenhill, J.; Street, R.A.; Dominik, M.; Sumi, T.; Szymański, M.K.; et al. Frequency of Solar-like Systems and of Ice and Gas Giants Beyond the Snow Line from High-magnification Microlensing Events in 2005-2008. Astrophys. J. 2010, 720, 1073-1089. [CrossRef]

32. Howard, A.W.; Marcy, G.W.; Bryson, S.T.; Jenkins, J.M.; Rowe, J.F.; Batalha, N.M.; Borucki, W.J.; Koch, D.G.; Dunham, E.W.; Gautier, T.N., III; et al. Planet Occurrence within 0.25 AU of Solar-type Stars from Kepler. Astrophys. J. Suppl. Ser. 2012, 201, 15. [CrossRef]

33. Mayor, M.; Marmier, M.; Lovis, C.; Udry, S.; Ségransan, D.; Pepe, F.; Benz, W.; Bertaux, J.; Bouchy, F.; Dumusque, X.; et al. The HARPS search for southern extra-solar planets XXXIV. Occurrence, mass distribution and orbital properties of super-Earths and Neptune-mass planets. arXiv 2011, arXiv:1109.2497.

34. Cassan, A.; Kubas, D.; Beaulieu, J.P.; Dominik, M.; Horne, K.; Greenhill, J.; Wambsganss, J.; Menzies, J.; Williams, A.; Jørgensen, U.G.; et al. One or more bound planets per Milky Way star from microlensing observations. Nature 2012, 481, 167-169. [CrossRef] [PubMed]

35. Burke, C.J.; Christiansen, J.L.; Mullally, F.; Seader, S.; Huber, D.; Rowe, J.F.; Coughlin, J.L.; Thompson, S.E.; Catanzarite, J.; Clarke, B.D.; et al. Terrestrial Planet Occurrence Rates for the Kepler GK Dwarf Sample. Astrophys. J. 2015, 809, 8. [CrossRef]

36. Suzuki, D.; Bennett, D.P.; Sumi, T.; Bond, I.A.; Rogers, L.A.; Abe, F.; Asakura, Y.; Bhattacharya, A.; Donachie, M.; Freeman, M.; et al. The Exoplanet Mass-ratio Function from the MOA-II Survey: Discovery of a Break and Likely Peak at a Neptune Mass. Astrophys. J. 2016, 833, 145. [CrossRef]

37. Laughlin, G.; Bodenheimer, P.; Adams, F.C. The Core Accretion Model Predicts Few Jovian-Mass Planets Orbiting Red Dwarfs. Astrophys. J. Lett. 2004, 612, L73-L76. [CrossRef]

38. Mordasini, C. Planetary population synthesis. arXiv 2018, arXiv:1804.01532

39. Clanton, C.; Gaudi, B.S. Synthesizing Exoplanet Demographics: A Single Population of Long-period Planetary Companions to M Dwarfs Consistent with Microlensing, Radial Velocity, and Direct Imaging Surveys. Astrophys. J. 2016, 819, 125. [CrossRef]

40. Shvartzvald, Y.; Maoz, D.; Udalski, A.; Sumi, T.; Friedmann, M.; Kaspi, S.; Poleski, R.; Szymański, M.K.; Skowron, J.; Kozłowski, S.; et al. The frequency of snowline-region planets from four years of OGLE-MOAWise second-generation microlensing. Mon. Not. R. Astron. Soc. 2016, 457, 4089-4113. [CrossRef]

41. Nataf, D.M.; Gould, A.; Fouqué, P.; Gonzalez, O.A.; Johnson, J.A.; Skowron, J.; Udalski, A.; Szymański, M.K.; Kubiak, M.; Pietrzyński, G.; et al. Reddening and Extinction toward the Galactic Bulge from OGLE-III: The Inner Milky Way's $\mathrm{R}_{V} \sim 2.5$ Extinction Curve. Astrophys. J. 2013, 769, 88. [CrossRef]

42. Boyajian, T.S.; von Braun, K.; van Belle, G.; Farrington, C.; Schaefer, G.; Jones, J.; White, R.; McAlister, H.A.; ten Brummelaar, T.A.; Ridgway, S.; et al. Stellar Diameters and Temperatures. III. Main Sequence A, F, G, and K Stars: Additional High-precision Measurements and Empirical Relations. Astrophys. J. 2013, 771, 40. Erratum in 2014, 787, 92. [CrossRef]

43. Kervella, P.; Thévenin, F.; Di Folco, E.; Ségransan, D. The angular sizes of dwarf stars and subgiants. Surface brightness relations calibrated by interferometry. Astron. Astrophys. 2004, 426, 297-307. [CrossRef] 
44. Gould, A. Extending the MACHO search to about 10 exp 6 solar masses. Astrophys. J. 1992, 392, $442-451$. [CrossRef]

45. Gould, A.; Udalski, A.; Monard, B.; Horne, K.; Dong, S.; Miyake, N.; Sahu, K.; Bennett, D.P.; Wyrzykowski, Ł.; Soszyński, I.; et al. The Extreme Microlensing Event OGLE-2007-BLG-224: Terrestrial Parallax Observation of a Thick-Disk Brown Dwarf. Astrophys. J. Lett. 2009, 698, L147-L151. [CrossRef]

46. Calchi Novati, S.; Gould, A.; Udalski, A.; Menzies, J.W.; Bond, I.A.; Shvartzvald, Y.; Street, R.A.; Hundertmark, M.; Beichman, C.A.; Yee, J.C.; et al. Pathway to the Galactic Distribution of Planets: Combined Spitzer and Ground-Based Microlens Parallax Measurements of 21 Single-Lens Events. Astrophys. J. 2015, 804, 20. [CrossRef]

47. Shvartzvald, Y.; Li, Z.; Udalski, A.; Gould, A.; Sumi, T.; Street, R.A.; Calchi Novati, S.; Hundertmark, M.; Bozza, V.; Beichman, C.; et al. The First Simultaneous Microlensing Observations by Two Space Telescopes: Spitzer and Swift Reveal a Brown Dwarf in Event OGLE-2015-BLG-1319. Astrophys. J. 2016, 831, 183. [CrossRef]

48. Henderson, C.B.; Poleski, R.; Penny, M.; Street, R.A.; Bennett, D.P.; Hogg, D.W.; Gaudi, B.S.; K2 Campaign 9 Microlensing Science Team.; Zhu, W.; Barclay, T.; et al. Campaign 9 of the K2 Mission: Observational Parameters, Scientific Drivers, and Community Involvement for a Simultaneous Space- and Ground-based Microlensing Survey. Publ. Astron. Soc. Pac. 2016, 128, 124401. [CrossRef]

49. Udalski, A.; Yee, J.C.; Gould, A.; Carey, S.; Zhu, W.; Skowron, J.; Kozłowski, S.; Poleski, R.; Pietrukowicz, P.; Pietrzyński, G.; et al. Spitzer as a Microlens Parallax Satellite: Mass Measurement for the OGLE-2014-BLG-0124L Planet and its Host Star. Astrophys. J. 2015, 799, 237. [CrossRef]

50. Beaulieu, J.P.; Batista, V.; Bennett, D.P.; Marquette, J.B.; Blackman, J.W.; Cole, A.A.; Coutures, C.; Danielski, C.; Dominis Prester, D.; Donatowicz, J.; et al. Combining Spitzer Parallax and Keck II Adaptive Optics Imaging to Measure the Mass of a Solar-like Star Orbited by a Cold Gaseous Planet Discovered by Microlensing. Astron. J. 2018, 155, 78. [CrossRef]

51. Mogavero, F.; Beaulieu, J.P. Microlensing planet detection via geosynchronous and low Earth orbit satellites. Astron. Astrophys. 2016, 585, A62. [CrossRef]

52. Bennett, D.P.; Anderson, J.; Bond, I.A.; Udalski, A.; Gould, A. Identification of the OGLE-2003-BLG-235/ MOA-2003-BLG-53 Planetary Host Star. Astrophys. J. Lett. 2006, 647, L171-L174. [CrossRef]

53. Delfosse, X.; Forveille, T.; Ségransan, D.; Beuzit, J.L.; Udry, S.; Perrier, C.; Mayor, M. Accurate masses of very low mass stars. IV. Improved mass-luminosity relations. Astrophys. J. 2000, 364, 217-224.

54. Bertelli, G.; Girardi, L.; Marigo, P.; Nasi, E. Scaled solar tracks and isochrones in a large region of the Z-Y plane. I. From the ZAMS to the TP-AGB end for 0.15-2.5 $M_{\odot}$ stars. Astron. Astrophys. 2008, 484, 815-830. [CrossRef]

55. Batista, V.; Beaulieu, J.P.; Bennett, D.P.; Gould, A.; Marquette, J.B.; Fukui, A.; Bhattacharya, A. Confirmation of the OGLE-2005-BLG-169 Planet Signature and Its Characteristics with Lens-Source Proper Motion Detection. Astrophys. J. 2015, 808, 170. [CrossRef]

56. Bennett, D.P.; Bhattacharya, A.; Anderson, J.; Bond, I.A.; Anderson, N.; Barry, R.; Batista, V.; Beaulieu, J.P.; DePoy, D.L.; Dong, S.; et al. Confirmation of the Planetary Microlensing Signal and Star and Planet Mass Determodellingations for Event OGLE-2005-BLG-169. Astrophys. J. 2015, 808, 169. [CrossRef]

57. Koshimoto, N.; Shvartzvald, Y.; Bennett, D.P.; Penny, M.T.; Hundertmark, M.; Bond, I.A.; Zang, W.C.; Henderson, C.B.; Suzuki, D.; Rattenbury, N.J.; et al. MOA-2016-BLG-227Lb: A Massive Planet Characterized by Combining Light-curve Analysis and Keck AO Imaging. Astron. J. 2017, 154, 3. [CrossRef]

58. Batista, V.; Beaulieu, J.P.; Bennett, D.P.; Marquette, J.B. OGLE-2013-BLG-132. Astrophys. J. 2018, submitted.

59. Beaulieu, J.P.E.A. AO. Astrophys. J. 2018, submitted.

60. Batista, V.; Gould, A.; Dieters, S.; Dong, S.; Bond, I.; Beaulieu, J.P.; Maoz, D.; Monard, B.; Christie, G.W.; McCormick, J.; et al. MOA-2009-BLG-387Lb: a massive planet orbiting an M dwarf. Astron. Astrophys. 2011, 529, A102. [CrossRef]

61. Gould, A. Resolution of the MACHO-LMC-5 Puzzle: The Jerk-Parallax Microlens Degeneracy. Astrophys. J. 2004, 606, 319-325. [CrossRef]

62. Alcock, C.; Allsman, R.A.; Alves, D.R.; Axelrod, T.S.; Becker, A.C.; Bennett, D.P.; Cook, K.H.; Drake, A.J.; Freeman, K.C.; Geha, M.; et al. Direct detection of a microlens in the Milky Way. Nature 2001, 414, 617-619. [CrossRef] [PubMed] 
63. Gould, A.; Udalski, A.; An, D.; Bennett, D.P.; Zhou, A.Y.; Dong, S.; Rattenbury, N.J.; Gaudi, B.S.; Yock, P.C.M.; Bond, I.A.; et al. Microlens OGLE-2005-BLG-169 Implies That Cool Neptune-like Planets Are Common. Astrophys. J. Lett. 2006, 644, L37-L40. [CrossRef]

64. Minniti, D.; Lucas, P.W.; Emerson, J.P.; Saito, R.K.; Hempel, M.; Pietrukowicz, P.; Ahumada, A.V.; Alonso, M.V.; Alonso-Garcia, J.; Arias, J.I.; et al. VISTA Variables in the Via Lactea (VVV): The public ESO near-IR variability survey of the Milky Way. New Astron. 2010, 15, 433-443. [CrossRef]

65. Bertin, E. PSFEx: Point Spread Function Extractor; Astrophysics Source Code Library: Los Alamos, NM, USA, 2013.

66. Bertin, E.; Arnouts, S. SExtractor: Software for source extraction. Astron. Astrophys. Suppl. Ser. 1996, 117, 393-404. [CrossRef]

67. Bertin, E. SCAMP: Automatic Astrometric and Photometric Calibration; Astrophysics Source Code Library: Los Alamos, NM, USA, 2010.

68. Bertin, E. SWarp: Resampling and Co-adding FITS Images Together; Astrophysics Source Code Library: Los Alamos, NM, USA, 2010.

69. Taylor, M.B. TOPCAT, STIL: Starlink Table/VOTable Processing Software. In Astronomical Data Analysis Software and Systems XIV; Shopbell, P., Britton, M.; Ebert, R., Eds.; Astronomical Society of the Pacific: San Francisco, CA, USA, 2005; Volume 347, p. 29.

70. Witzel, G.; Lu, J.R.; Ghez, A.M.; Martinez, G.D.; Fitzgerald, M.P.; Britton, M.; Sitarski, B.N.; Do, T.; Campbell, R.D.; Service, M.; et al. The AIROPA software package: milestones for testing general relativity in the strong gravity regime with AO. Adapt. Opt. Syst. V SPIE 2016, 9909, 990910.

71. Gonzalez, O.A.; Rejkuba, M.; Zoccali, M.; Valenti, E.; Minniti, D.; Schultheis, M.; Tobar, R.; Chen, B. Reddening and metallicity maps of the Milky Way bulge from VVV and 2MASS. II. The complete high resolution extinction map and implications for Galactic bulge studies. Astron. Astrophys. 2012, 543, A13. [CrossRef]

72. Nishiyama, S.; Tamura, M.; Hatano, H.; Kato, D.; Tanabé, T.; Sugitani, K.; Nagata, T. Interstellar Extinction Law Toward the Galactic Center III: J, H, $\mathrm{K}_{S}$ Bands in the 2MASS and the MKO Systems, and 3.6, 4.5, 5.8, $8.0 \mu \mathrm{m}$ in the Spitzer/IRAC System. Astrophys. J. 2009, 696, 1407-1417. [CrossRef]

73. Santerne, A.; Beaulieu, J.P.; Rojas Ayala, B.; Boisse, I.; Schlawin, E.; Almenara, J.M.; Batista, V.; Bennett, D.; Díaz, R.F.; Figueira, P.; et al. Spectroscopic characterisation of microlensing events. Towards a new interpretation of OGLE-2011-BLG-0417. Astron. Astrophys. 2016, 595, L11. [CrossRef]

74. Shvartzvald, Y.; Calchi Novati, S.; Gaudi, B.S.; Bryden, G.; Nataf, D.M.; Penny, M.T.; Beichman, C.; Henderson, C.B.; Jacklin, S.; et al. UKIRT-2017-BLG-001Lb: A giant planet detected through the dust. arXiv 2018, arXiv:1802.06795.

75. Yee, J.C.; Han, C.; Gould, A.; Skowron, J.; Bond, I.A.; Udalski, A.; Hundertmark, M.; Monard, L.A.G.; Porritt, I.; Nelson, P.; et al. MOA-2013-BLG-220Lb: Massive Planetary Companion to Galactic-disk Host. Astrophys. J. 2014, 790, 14. [CrossRef]

76. Mróz, P.; Udalski, A.; Bond, I.A.; Skowron, J.; Sumi, T.; Han, C.; Szymański, M.K.; Soszyński, I.; Poleski, R.; Pietrukowicz, P.; et al. OGLE-2013-BLG-0132Lb and OGLE-2013-BLG-1721Lb: Two Saturn-mass Planets Discovered around M-dwarfs. Astron. J. 2017, 154, 205. [CrossRef]

77. Han, C.; Udalski, A.; Choi, J.Y.; Yee, J.C.; Gould, A.; Christie, G.; Tan, T.G.; Szymański, M.K.; Kubiak, M.; Soszyński, I.; et al. The Second Multiple-planet System Discovered by Microlensing: OGLE-2012-BLG-0026Lbc, A Pair of Jovian Planets beyond the Snow Line. Astrophys. J. Lett. 2013, 762, L28. [CrossRef]

78. Yee, J.C.; Shvartzvald, Y.; Gal-Yam, A.; Bond, I.A.; Udalski, A.; Kozłowski, S.; Han, C.; Gould, A.; Skowron, J.; Suzuki, D.; et al. MOA-2011-BLG-293Lb: A Test of Pure Survey Microlensing Planet Detections. Astrophys. J. 2012, 755, 102. [CrossRef]

79. Dong, S.; Bond, I.A.; Gould, A.; Kozłowski, S.; Miyake, N.; Gaudi, B.S.; Bennett, D.P.; Abe, F.; Gilmore, A.C.; Fukui, A.; et al. Microlensing Event MOA-2007-BLG-400: Exhuming the Buried Signature of a Cool, Jovian-Mass Planet. Astrophys. J. 2009, 698, 1826-1837. [CrossRef]

80. Udalski, A.; Jaroszyński, M.; Paczyński, B.; Kubiak, M.; Szymański, M.K.; Soszyński, I.; Pietrzyński, G.; Ulaczyk, K.; Szewczyk, O.; Wyrzykowski, Ł.; et al. A Jovian-Mass Planet in Microlensing Event OGLE-2005-BLG-071. Astrophys. J. Lett. 2005, 628, L109-L112. [CrossRef] 
81. Dong, S.; Gould, A.; Udalski, A.; Anderson, J.; Christie, G.W.; Gaudi, B.S.; OGLE Collaboration; Jaroszyński, M.; Kubiak, M.; Szymański, M.K.; et al. OGLE-2005-BLG-071Lb, the Most Massive M Dwarf Planetary Companion? Astrophys. J. 2009, 695, 970-987. [CrossRef]

82. Bond, I.A.; Udalski, A.; Jaroszyński, M.; Rattenbury, N.J.; Paczyński, B.; Soszyński, I.; Wyrzykowski, L.; Szymański, M.K.; Kubiak, M.; Szewczyk, O.; et al. OGLE 2003-BLG-235/MOA 2003-BLG-53: A Planetary Microlensing Event. Astrophys. J. Lett. 2004, 606, L155-L158. [CrossRef]

83. Koshimoto, N.; Udalski, A.; Beaulieu, J.P.; Sumi, T.; Bennett, D.P.; Bond, I.A.; Rattenbury, N.; Fukui, A.; Batista, V.; Marquette, J.B.; et al. OGLE-2012-BLG-0950Lb: The First Planet Mass Measurement from Only Microlens Parallax and Lens Flux. Astron. J. 2017, 153, 1. [CrossRef]

84. Fukui, A.; Gould, A.; Sumi, T.; Bennett, D.P.; Bond, I.A.; Han, C.; Suzuki, D.; Beaulieu, J.P.; Batista, V.; Udalski, A.; et al. OGLE-2012-BLG-0563Lb: A Saturn-mass Planet around an M Dwarf with the Mass Constrained by Subaru AO Imaging. Astrophys. J. 2015, 809, 74. [CrossRef]

85. Poleski, R.; Udalski, A.; Bond, I.A.; Beaulieu, J.P.; Clanton, C.; Gaudi, S.; Szymański, M.K.; Soszyński, I.; Pietrukowicz, P.; Kozłowski, S.; et al. A companion on the planet/brown dwarf mass boundary on a wide orbit discovered by gravitational microlensing. arXiv 2017, arXiv:1704.01121.

86. Cole, A.; Beaulieu, J.P.; Marquette, J.B. OGLE-2015-BLG-1395. Astrophys. J. 2018, submitted.

87. Yee, J.C.; Gould, A.; Beichman, C.; Calchi Novati, S.; Carey, S.; Gaudi, B.S.; Henderson, C.B.; Nataf, D.; Penny, M.; Shvartzvald, Y.; Zhu, W. Criteria for Sample Selection to Maximize Planet Sensitivity and Yield from Space-Based Microlens Parallax Surveys. Astrophys. J. 2015, 810, 155. [CrossRef]

88. Yee, J.C.; Fazio, G.G.; Benjamin, R.; Kirkpatrick, J.D.; Malkan, M.A.; Trilling, D.; Carey, S.; Ciardi, D.R.; Apai, D.; Ashby, M.L.N.; et al. The Science Case for an Extended Spitzer Mission. arXiv 2017, arXiv:1710.04194.

(C) 2018 by the authors. Licensee MDPI, Basel, Switzerland. This article is an open access article distributed under the terms and conditions of the Creative Commons Attribution (CC BY) license (http://creativecommons.org/licenses/by/4.0/). 